\title{
UV initiated thiol-ene chemistry: a facile and modular synthetic methodology for the construction of functional 3D networks with tunable properties $\uparrow$
}

Cite this: J. Mater. Chem. A, 2013, 1, 13732

Received 29th July 2013

Accepted 26th September 2013

DOI: $10.1039 / c 3 t a 12963 b$

\author{
Surinthra Mongkhontreerat, ${ }^{a}$ Kim Öberg, ${ }^{a}$ Lina Erixon, ${ }^{a}$ Peter Löwenhielm, ${ }^{\text {b }}$ \\ Anders Hult ${ }^{a}$ and Michael Malkoch ${ }^{* a}$
}

www.rsc.org/MaterialsA

\begin{abstract}
A facile methodology for the fabrication of functional crosslinked three dimensional (3D) networks has herein been explored via the benign and UV initiated thiol-ene coupling (TEC) chemistry. The careful selection of monomers or polymers and their feed ratio resulted in straightforward design of organic, inorganic and hydrogel networks with readily available alkenes or thiol functional groups. All crosslinked networks were fabricated within 1 second of UV exposure at wavelengths of 320-390 nm and generally exhibited excellent gel fractions around $90 \%$. By introducing off-stoichiometric thiol and ene (OSTE) monomer feed ratios the window of mechanical properties could be manipulated. For the organic triazine system, the Young's modulus was altered from $780 \mathrm{MPa}$ at an equimolar monomer ratio to soft $106 \mathrm{kPa}$ for 2.5 equiv. with excess of thiol compared to enes. Postfunctionalizations with hydrophilic polyethylene glycols or acrylic acid and hydrophobic heneicosafluorododecyl acrylate were explored for the manipulation of functional networks. In this case, the rigid networks with excess of thiols were used as model substrates of which the initial contact angle (CA) of $60^{\circ}$ was decreased to $43^{\circ}$ by the introduction of acrylic acid and increased to $140^{\circ}$ by successful attachment of fluorinated molecules. Finally, amalgamating micropatterning strategy with simple postfunctionalizations of hydrophobic groups resulted in superhydrophobic rigid surfaces with a CA of $173^{\circ}$.
\end{abstract}

The understanding of cell activities and behaviors in solution or on substrates is paramount for application driven research in therapeutic and diagnostic fields. For reasonable and accurate investigations of cell interaction mechanisms, the design of tunable 3D networks plays today an important role. In sensing

${ }^{a}$ KTH Royal Institute of Technology, School of Chemical Science and Engineering, Dept. of Fibre and Polymer Technology, SE-100 44, Stockholm, Sweden. E-mail: malkoch@ kth.se; Tel: +4687908768

${ }^{b} S P$ Technical Research Institute of Sweden, Box 857, SE 501 15, Borås, Sweden $\dagger$ Electronic supplementary information (ESI) available: Network formation, synthesis of precursor materials for post-functionalization, UV analysis/Ellman's reagent, DMA curves, calculation of $\left(\bar{M}_{\mathrm{c}}\right)$, network post-functionalizations, FT-IR spectra, and SEM images for the molding technique. See DOI: 10.1039/c3ta12963b applications, high detection resolution is strongly coupled to the optimization of the number of surface recognition sites and selectivity. ${ }^{1}$ From an accessibility point of view, the fabricated networks are preferably produced from commercial sources coupled with easy handling and excellent storage properties. Another important aspect is the mechanical properties of the networks, which allow enhanced cell adhesion. ${ }^{2}$

Crosslinked 3D networks with readily available functionalities for facile postfunctionalizations deliver an elegant modular alternative to explore for biological studies. The ability to topographically functionalize such surfaces with anticipated substituents allows their use in an array of desired applications. For instance, covalent attachment of hydrophilic and/or biological sensing molecules enables the assessment of $3 \mathrm{D}$ networks in immunoassay and bio-recognition applications or as molecular imprinted networks. ${ }^{3}$ Additionally, in a number of biological studies in which small capillary channels are used as devices, the Young's modulus $(E)$ window of the networks needs to be low enough for microfabrication but sufficiently high to maintain the geometry during low pressure operations. Typically, for PDMS networks the Young's modulus range is between $0.5 \mathrm{MPa}$ and $5 \mathrm{MPa}^{4,5}$ However, in cell culture, soft surfaces with a low modulus are preferable for efficient cell adhesion. ${ }^{2}$ Consequently, novel materials with tuneable modulus and programmable functionalities are continuously in demand in these fields.

Commonly, microarrays for biological studies and sensing applications are fabricated using commercial polydimethylsiloxane (PDMS) (Sylgard 184). ${ }^{6}$ Even though PDMS is robust and widely used, there are several limitations including (i) long thermal curing processes, ${ }^{7}$ (ii) inherent hydrophobic nature, ${ }^{6,8}$ (iii) chemical inertness in which plasmarization is required to introduce short-term hydrophilicity ${ }^{\mathbf{9 , 1 0}}$ and hydroxyl functional groups for further chemical modification, ${ }^{7,8}$ and (iv) the geometry deformation which occurs under low pressure operation due to the low modulus. ${ }^{11}$ Considering the number of criteria of functional surfaces, a modular approach is herein exploited using UV initiated thiol-ene coupling (TEC) chemistry for the 
construction of functional 3D networks. The TEC chemistry has been extensively used due to the straightforwardness, robustness and the possibility to obtain high conversion, homogenous networks under ambient conditions and in the presence of oxygen. ${ }^{12,13}$ In contrast to the typical strategies relying on stoichiometrically balanced component ratios, for the development of neutral networks with maximized mechanical properties, the proposed methodology capitalizes on the off-stoichiometric ratio between thiol and ene (OSTE) monomers or polymers hence generating crosslinked functional 3D scaffolds with reactive sites available for further chemical modification. ${ }^{\mathbf{4}}$

Subsequently, we demonstrate the construction of imbalanced 3D networks via TEC chemistry covering a wide range of modulus and based on organic and inorganic compounds in the form of three different network platforms each possessing unique properties. The monomer/polymer feed ratios as well as a selection of properties for these networks are detailed in Fig. 1 and Table 1. The first set of networks were fabricated using triallyltriazinetrione (TTT) (1) and tris[2-(3-mercaptopropionyloxy)ethyl]isocyanurate (TMI) (2) as monomers. Six highly crosslinked networks with great stiffness, R1-R6, were obtained. In contrast to the rigid systems, the fabrication of soft functional networks that were inherently hydrophilic or hydrophobic was also sought out. In the second network, by mixing allyl functionalized hydrophilic polyethylene glycol (PEG $3 \mathrm{kDa}$ ) (3) with a pentaerythritol tetraki-mercaptopropionate (PTM) (4) crosslinker at $33 \mathrm{wt} \%$ solids in ethanol, highly covalently crosslinked hydrogels exhibiting high water swelling capacity were successfully fabricated. For both the rigid triazine and hydrogel systems, $0.58 \mathrm{wt} \%$ of 2,2-dimethoxy-2-phenylacetophenone (DMPA) was used as a photoinitiator. Soft hydrophobic PDMS networks with high flexibility and ductility were fabricated from vinyldimethylsiloxy terminated polydimethylsiloxane (5) (molecular weight $=28000 \mathrm{~g} \mathrm{~mol}^{-1}$ ) and poly(mercaptopropyl) methylsiloxane (6) (molecular weight $=$ 4000-7000 $\mathrm{g} \mathrm{mol}^{-1}$ ) without the presence of photoinitiator. In all cases, the 3D networks were accomplished within 0.5 second total UV exposure at wavelengths of 320-390 nm and an intensity of $184 \mathrm{~mW} \mathrm{~cm}^{-2}$. To investigate the network formation and the presence of unreacted functional groups, Fourier Transform RAMAN Spectroscopy (FT-RAMAN) was performed on a series of rigid networks. The spectra revealed $\mathrm{C}=\mathrm{C}$ stretching at $1656 \mathrm{~cm}^{-1}$ representing excess reactive allyl groups (R1 and R2) and $\mathrm{S}-\mathrm{H}$ stretching at $2564 \mathrm{~cm}^{-1}$ representing excess reactive thiol groups (R4-R6) (Fig. 2). Notably, network R3 with a balanced stoichiometric ratio of allyls to thiols displayed full consumption of both reactive groups. FT-RAMAN evaluation of excess functional groups on both hydrogel and PDMS 3D networks was uninformative due to the low end group concentration in comparison to the chain length of the prepolymers. To visualize the controlled introduction of available thiol groups, all the networks were corroborated by exploiting allyloxy disperse red 13 (7) as a visual colorimetric reagent. ${ }^{15}$ UV initiated postfunctionalization via thiol-ene click reactions ${ }^{\mathbf{1 6 , 1 7}}$ of networks with surplus of thiols resulted in increased covalent attachment of the colorimetric reagent. As shown in Fig. 3, the

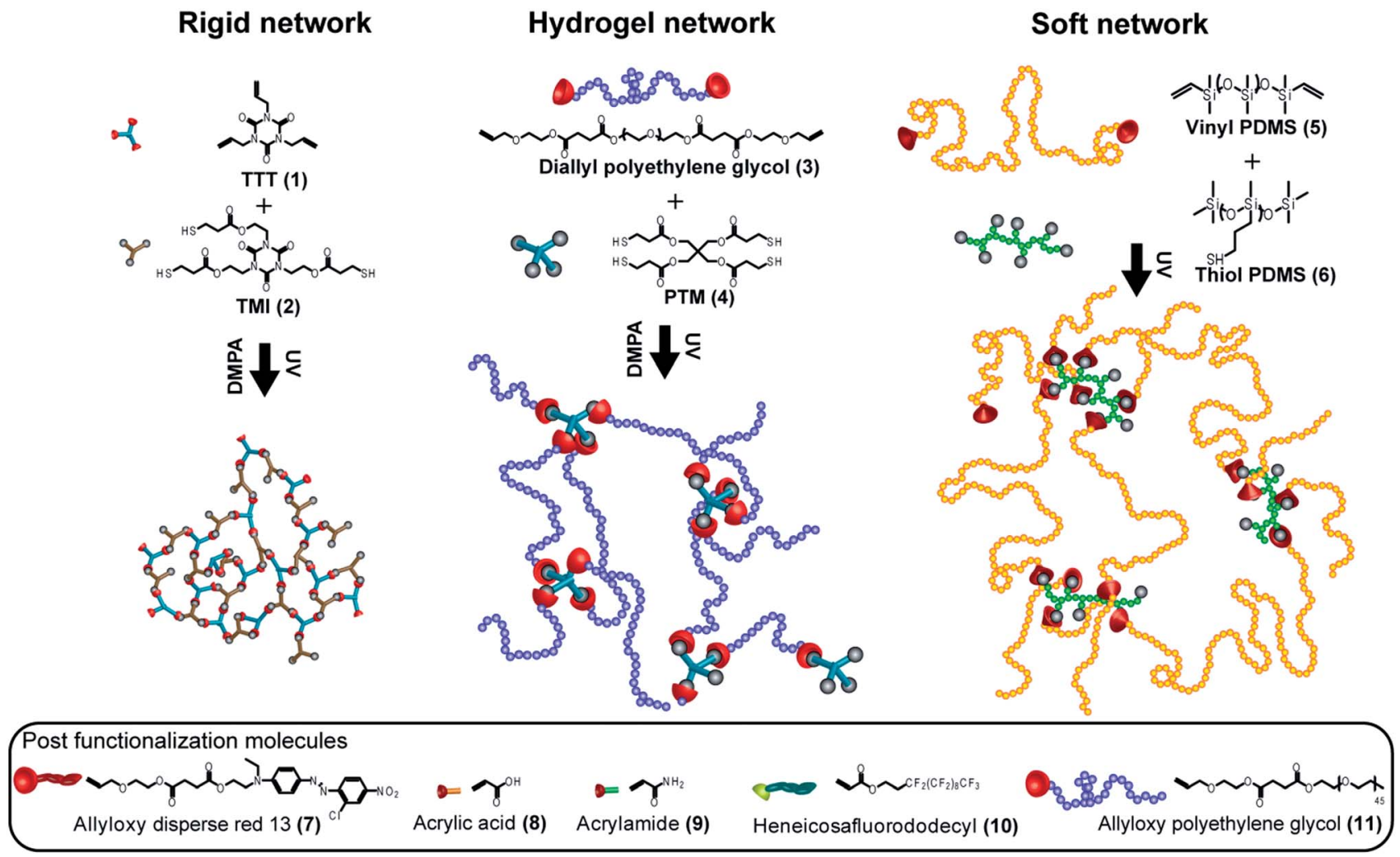

Fig. 1 Schematic representation of three different functional networks and the postfunctionalization molecules. 
Table 1 Mechanical properties of functional networks with a series of stoichiometric ratios

\begin{tabular}{|c|c|c|c|c|c|c|c|}
\hline & Sample code & Ene : thiol molar ratio & Young's modulus & $\bar{M}_{\mathrm{c}}\left(\mathrm{g} \mathrm{mol}^{-1}\right)^{a}$ & $T_{\mathrm{g}}\left({ }^{\circ} \mathrm{C}\right)$ & $\%$ swelling $^{b}$ & Gel fraction \\
\hline \multirow[t]{5}{*}{ Rigid network } & $\mathrm{R} 1$ & $1.9: 1$ & $540 \pm 48 \mathrm{MPa}$ & 1200 & 67 & $27 \pm 0.7$ & $95 \pm 0.4$ \\
\hline & $\mathrm{R} 2$ & $1.5: 1$ & $615 \pm 66 \mathrm{MPa}$ & 900 & 72 & $25 \pm 0.5$ & $98 \pm 0.3$ \\
\hline & $\mathrm{R} 4$ & $1: 1.5$ & $89 \pm 4 \mathrm{MPa}$ & 1700 & 48 & $29 \pm 1.0$ & $98 \pm 1.3$ \\
\hline & R5 & $1: 1.9$ & $659 \pm 26 \mathrm{kPa}$ & 4000 & 30 & $26 \pm 2.0$ & $87 \pm 0.0$ \\
\hline & R6 & $1: 2.5$ & $106 \pm 29 \mathrm{kPa}$ & 31000 & 24 & $11 \pm 0.5$ & $68 \pm 0.8$ \\
\hline & H3 & $1.5: 1$ & $18 \pm 4 \mathrm{kPa}$ & 1675 & N/A & $975 \pm 45$ & $66 \pm 0.9$ \\
\hline \multirow[t]{4}{*}{ Soft network } & $\mathrm{S} 1$ & $1: 2$ & $98 \pm 11 \mathrm{kPa}$ & 36000 & N/A & $1100 \pm 20$ & $80 \pm 0.5$ \\
\hline & S2 & $1: 3$ & $266 \pm 63 \mathrm{kPa}$ & 12000 & N/A & $750 \pm 6$ & $93 \pm 0.3$ \\
\hline & S3 & $1: 6$ & $185 \pm 27 \mathrm{kPa}$ & 17000 & N/A & $900 \pm 15$ & $92 \pm 0.3$ \\
\hline & $\mathrm{S} 4$ & $1: 9$ & $143 \pm 38 \mathrm{kPa}$ & 26000 & N/A & $1100 \pm 29$ & $89 \pm 0.5$ \\
\hline
\end{tabular}

${ }^{a} \bar{M}_{\mathrm{c}}$ was calculated from DMTA (rigid and soft networks) and swelling properties (hydrogel network). ${ }^{b}$ Swelling tests were performed in acetone (rigid network), water (hydrogel network) and chloroform (soft network).

increased excess of available thiols within the films resulted in higher degree of disperse red attachment which subsequently yielded stronger colouring outcome. Additionally, to quantify the available thiol groups after film formation, the networks were initially exposed to Ellman's reagent (5,5'-dithiobis-2nitrobenzoic acid (DTNB)) in phosphate buffer solution, $\mathrm{pH}$ $7{ }^{18,19}$ The reagent selectively reacts with thiol groups forming the yellow chromophore derivative, 5-thio-2-nitrobenzoic acid (TNB), which possesses high absorbance at $412 \mathrm{~nm}$. UV/vis analysis of TNB enables the determination of available thiols according to the absorbance calibration curve. ${ }^{18}$ The number of thiol molecules per cubic nanometer in hydrogel networks can be calculated by swelling hydrogels in DTNB solution. The hydrogel with balanced stoichiometric ratio $\mathrm{H} 1$ was found to include unreacted thiols of 0.019 molecules per $\mathrm{nm}^{3}$ while $\mathrm{H} 2$ with a $2: 1$ ratio of [SH] : [allyl] revealed increased thiol active sites of 0.031 and 0.015 molecules per $\mathrm{nm}^{3}$ for the network with excess of allyl $\mathrm{H} 3$ with a $1.5: 1$ ratio of [allyl] : [SH]. As shown in Fig. 3, the presence of unreacted thiol groups in hydrogel network $\mathrm{H} 1$ was corroborated by the colorimetric reagent (7). $\mathrm{H} 1$ and $\mathrm{H} 3$ revealed the available thiol groups even though they are designed based on the equimolar feed ratio and excess of allyl. This is probably due to the restriction from an allyl PEG chain entanglement under the film formation conditions which

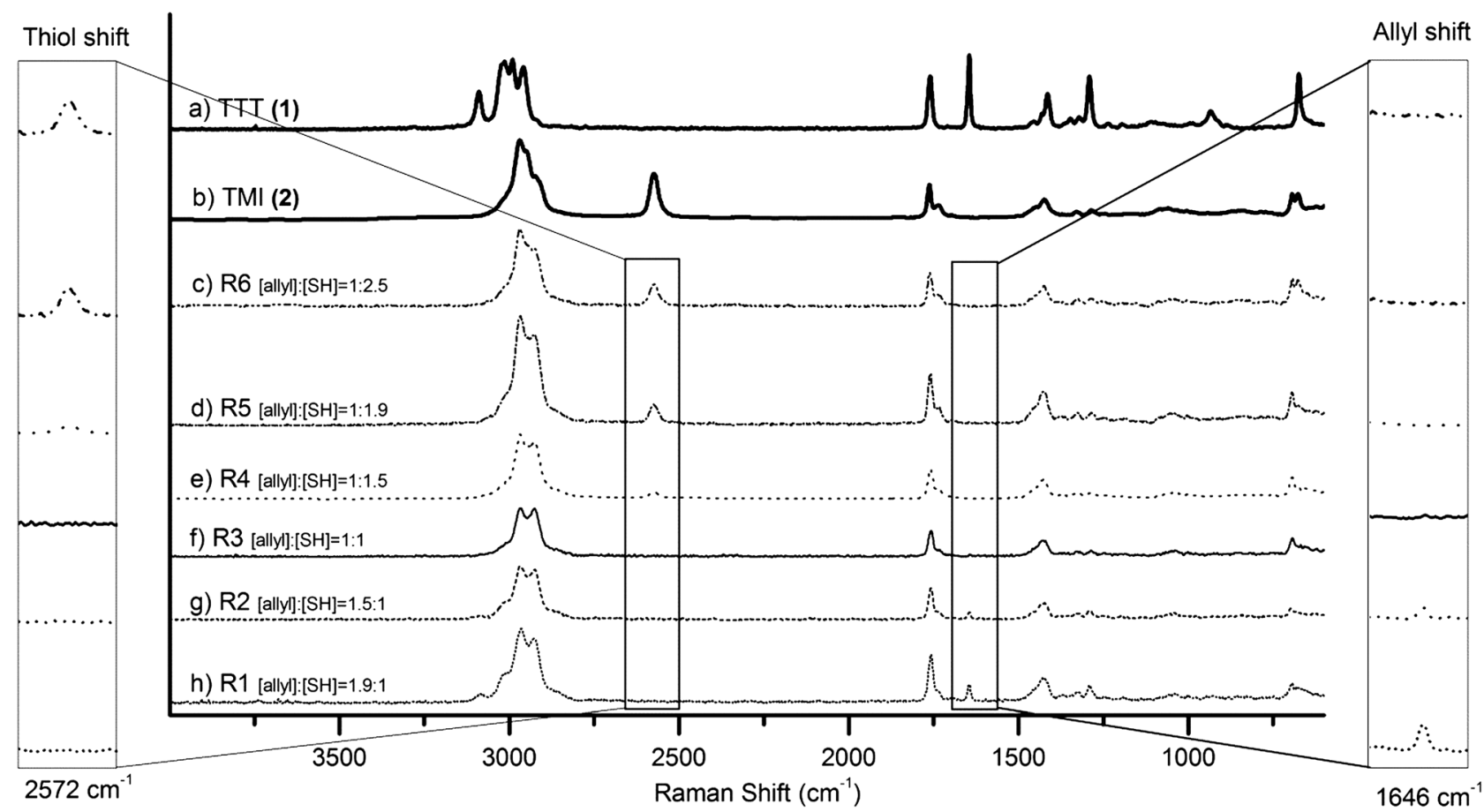

Fig. 2 FT-RAMAN spectra of a series of rigid networks and their monomers (a) allyl monomer (TTT (1)), (b) thiol monomer (TMI (2)), (c) R6, (d) R5, (e) R4, (f) R3, (g) R2, and (h) R1. 


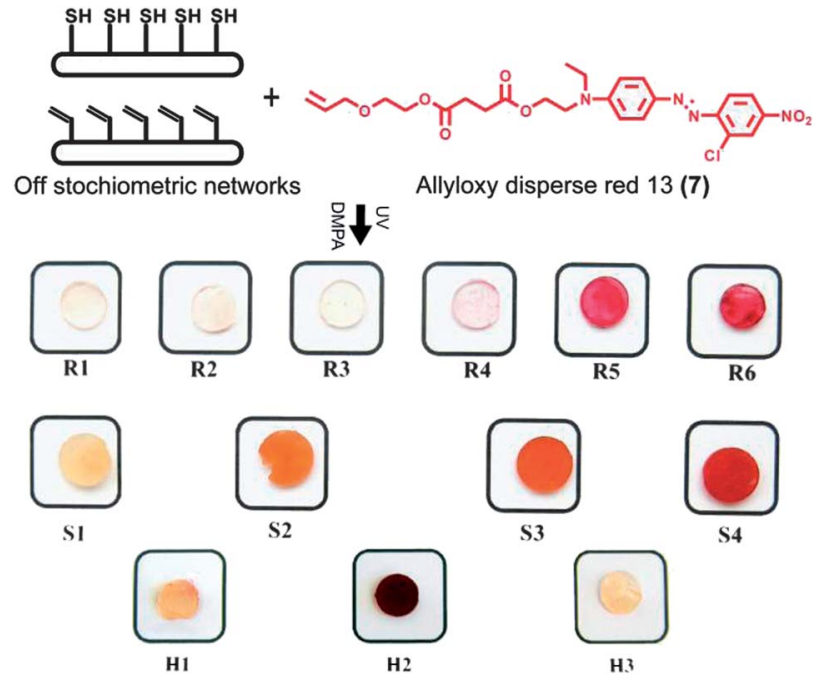

Fig. 3 Postfunctionalization with a visual colorimetric reagent allyloxy disperse red 13 (7) with increasing thiol surplus.

hinder the probability of the reaction of all allyl groups leading to some unreacted thiols.

Unfortunately, the sensitivity of thiol detection through Ellman's reagent was found insufficient for both rigid and soft inorganic networks. In this case, the limited network swelling with aqueous Ellman's solution results in unsuccessful interactions with excess thiols. Moreover, due to the hydrophobic nature and high mobility of the silicon networks, the thiol groups can be shielded for further reactions with the reagent under aqueous conditions. As seen in Table 1 and Fig. 4, the mechanical properties were controlled by altering both the composition of the components as well as the feed ratio between thiol and ene. For the rigid systems, the network with balance ratio R3 displayed the highest crosslinking density with a gel fraction close to $100 \%$, an average molecular weight between a crosslink $\bar{M}_{\mathrm{c}}$ of $800 \mathrm{~g} \mathrm{~mol}^{-1}$ and Young's modulus with a value above $780 \mathrm{MPa}$. The introduction of the off-stoichiometric strategy with unreacted functional groups resulted in looser networks with a higher value of $\bar{M}_{\mathrm{c}}$. Interestingly, a direct comparison between networks with excess of allyl or thiol with the same off stoichiometric ratio (R2 vs. R4) revealed similar gel fractions of $98 \%$ but with a lower $E$ value and higher $\bar{M}_{\mathrm{c}}$ for the networks with excess of thiol. In this case, the excess of allyl groups in R2 resulted in Young's modulus value of $615 \mathrm{MPa}$ and a $\bar{M}_{\mathrm{c}}$ of $900 \mathrm{~g} \mathrm{~mol}^{-1}$ while excess of thiol in R4 noted a Young's modulus value of $89 \mathrm{MPa}$ and $\bar{M}_{\mathrm{c}}$ of $1700 \mathrm{~g}$ $\mathrm{mol}^{-1}$. The apparent drop in mechanical properties may be due to the more flexible structure of the triazine thiol monomer or consumption of thiols forming spontaneous disulphide cyclization that does not participate during the crosslinking process. Moreover, the more rigid systems achieved for R2 can be a consequence of homo-crosslinking between the available allyls. It should be noted that the homopolymerization of triallyltriazinetrione (1) using similar conditions as thiol-ene networks resulted in viscous gel with a gel fraction of $2.6 \%$. Additional increase of thiols resulted in lower gel fractions reaching $68 \%$ at 2.5 molar ratio excess (R6). Notably, the obtained organic gels were found soft and loosely crosslinked, similar to the mechanical properties of commercially available inorganic PDMS networks, and with a Young's modulus value of $106 \mathrm{kPa}$ and $\bar{M}_{\mathrm{c}}$ of $31000 \mathrm{~g} \mathrm{~mol}^{-1}$.

In the case of soft networks, the inorganic system S2 shows the lowest $\bar{M}_{\mathrm{c}}$ of $12000 \mathrm{~g} \mathrm{~mol}^{-1}$ and swelling degree in chloroform as well as the highest gel fraction of $89 \%$ and Young's modulus of $266 \mathrm{kPa}$.

For hydrogels, both the balance ratio (H1) and excess of thiol (H2) have similar swelling/leaching properties in water compared to excess of allyl (H3) which can be seen from H3's lower gel fraction. Young's modulus and values of $\bar{M}_{\mathrm{c}}$ estimated from stiffness properties of the hydrogel network are practically similar. Satisfied with the modular strategy that delivers functional surfaces covering a range of modulus, the films were further exploited as templating scaffolds to manipulate their final properties by facile chemical modifications of readily available functional groups. In this context, the available thiols were approached for postfunctionalization purposes as these can efficiently react with a number of complementary reactive

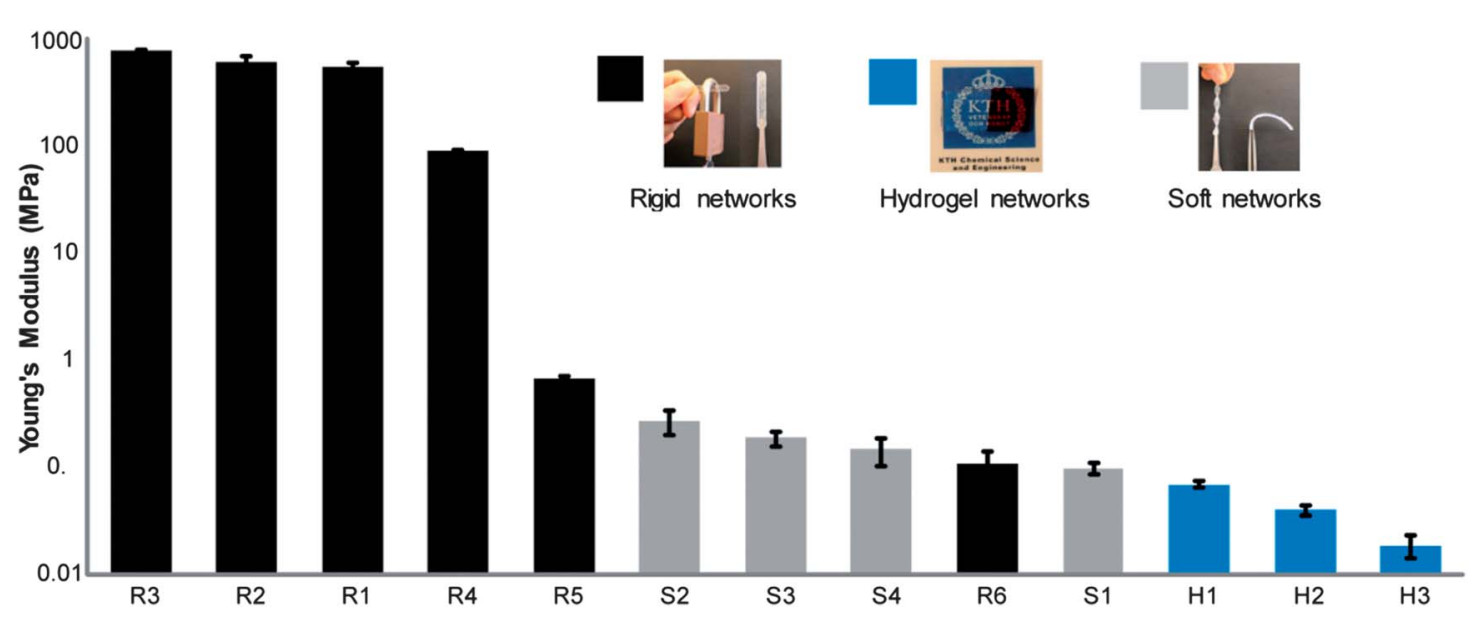

Fig. 4 Young's modulus of the functional network at room temperature $\left(25^{\circ} \mathrm{C}\right)$. 
groups including alkenes and alkynes, nucleophilic addition to epoxies and isocyanates, or through Michael addition. ${ }^{20}$ Following a similar solution based modification strategy as for the attachment of the colorimetric reagent (7) i.e. the robust and UV-initiated TEC click reaction, ${ }^{17}$ the substrates were conceptually postfunctionalized to deliver hydrophilic surfaces using acrylic acid (8), acrylamide (9) and allyloxy polyethylene glycol (11) as well as hydrophobicity by the attachment of the fluorinated compound, heneicosafluorododecyl acrylate (10).

While the functionalizations of the rigid networks were topological, the soft inorganic and hydrogel networks were homogenously modified. The latter was accomplished by swelling the networks in a mixture of reactive vinylic molecules and catalytic amounts of DMPA and followed by UV initiated TEC reactions. For the rigid substrates, R5 based on the 1.9 offstoichiometric amount of thiols was chosen as a scaffolding surface due to the moderate amount of unreacted thiol and robust mechanical properties. As can be seen in Fig. 5, the initial contact angle (CA) for the R5 film was noted at $60^{\circ}$. After surface modifications with a solution of acrylic acid (8) or allyloxy PEG (11), at UV exposure less than 1 second with a dosage of $372 \mathrm{~mJ}$ $\mathrm{cm}^{-2}$, followed by thorough washing procedures, the films displayed increased wettability with a contact angle decrease to $43^{\circ}$ and $52^{\circ}$, respectively. To alter the surface properties to exhibit hydrophobicity, heneicosafluorododecyl acrylate (10) was allowed to react with the $\mathrm{R} 5$ film which resulted in an increase of $80^{\circ}$ to a CA of $140^{\circ}$. For the soft hydrogels and inorganic systems, acrylic acid (8) and acrylamide (9) were efficiently attached to H2 and S3 networks. After solvent wash of the crosslinked system, FT-IR spectra (ESI S4 and S5 $\dagger$ ) showed the presence of $\mathrm{N}-\mathrm{H}$ stretching at $1608 \mathrm{~cm}^{-1}$ and $\mathrm{C}=\mathrm{O}$ at $1666 \mathrm{~cm}^{-1}$ for the acrylamide substituent. Similarly, the attachment of acrylic acid for the soft PDMS network shows the $\mathrm{C}=\mathrm{O}$ stretching at $1711 \mathrm{~cm}^{-1}$ as well as an increased peak for the hydrogel $\mathrm{C}=\mathrm{O}$ stretching when compared to the reference PEG network. In the case of soft
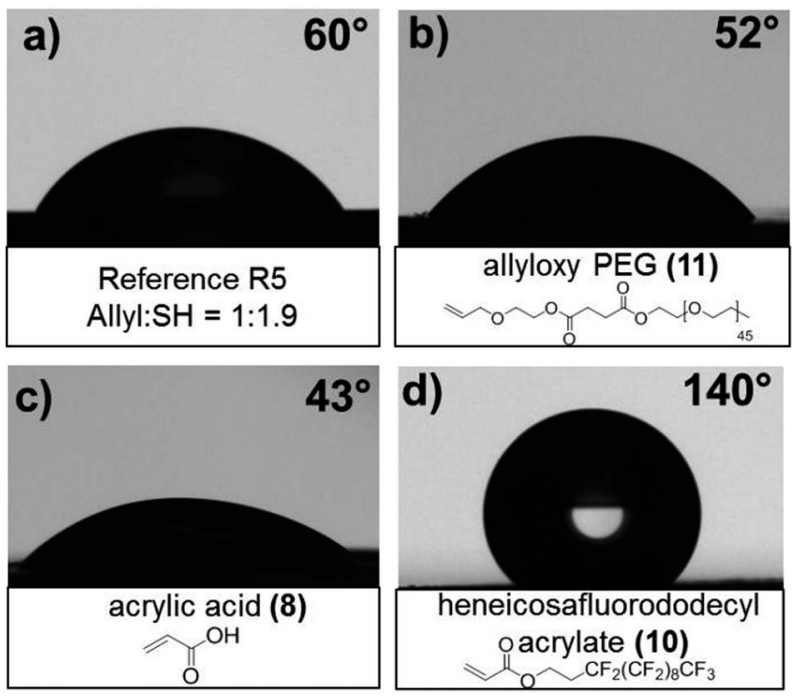

Fig. 5 Contact angle measurement of the rigid network (a) reference sample R5 and postfunctionalization with (b) allyloxy PEG (11), (c) acrylic acid (8) and (d) heneicosafluorododecyl acrylate (10).
PDMS based networks, the contact angle measurements did not display any decrease. This is due to the mobility of PDMS that migrates from the bulk to the surface resulting in hydrophobic recovery. ${ }^{9}$ Nonetheless, the attachment of the colorimetric reagent (7) resulted in a strong visual signal corroborating successful covalent postfunctionalization (Fig. 3). To further expand on the feasibility of the proposed methodology, microfabrication via the moulding technique ${ }^{21}$ was utilized to generate functional surfaces with increased topographic roughness and hydrophobicity. ${ }^{22}$

An attractive field of research capitalizes on superhydrophobic substrates that exhibit repelling properties with $\mathrm{CA}$ around $150^{\circ}$ that are in need in numbers of applications. Such surfaces find use as self-cleaning paints and textiles ${ }^{22}$ and in emerging applications such as DNA microarrays requiring specific wetting of the substrate. ${ }^{22}$ Both rigid (R5) and soft (S3) networks were patterned utilizing submicron ridge/groove silicon master structures $(10 \mu \mathrm{m})$, ESI Fig. S6. $\dagger$ The effect of surface topology raises the CA for the PDMS substrates (S3) from $120^{\circ}$ to superhydrophobic $149^{\circ}$ (Fig. 6a and b). Increasing the roughness on rigid networks (R5) resulted in an increase of CA to $120^{\circ}$ (Fig. 6c and d). Remarkably, amalgamating surface patterning of the rigid thiol functional substrate (R5) and postfunctionalization procedures with heneicosafluorododecyl acrylate (10) resulted in the superhydrophobic surface that displayed a static CA of $173^{\circ}$ based on $10 \mu$ l water droplets (Fig. 6e). In fact, due to strong repulsion forces the contact angle measurements with water droplets of $5 \mu \mathrm{l}$ were fruitless.
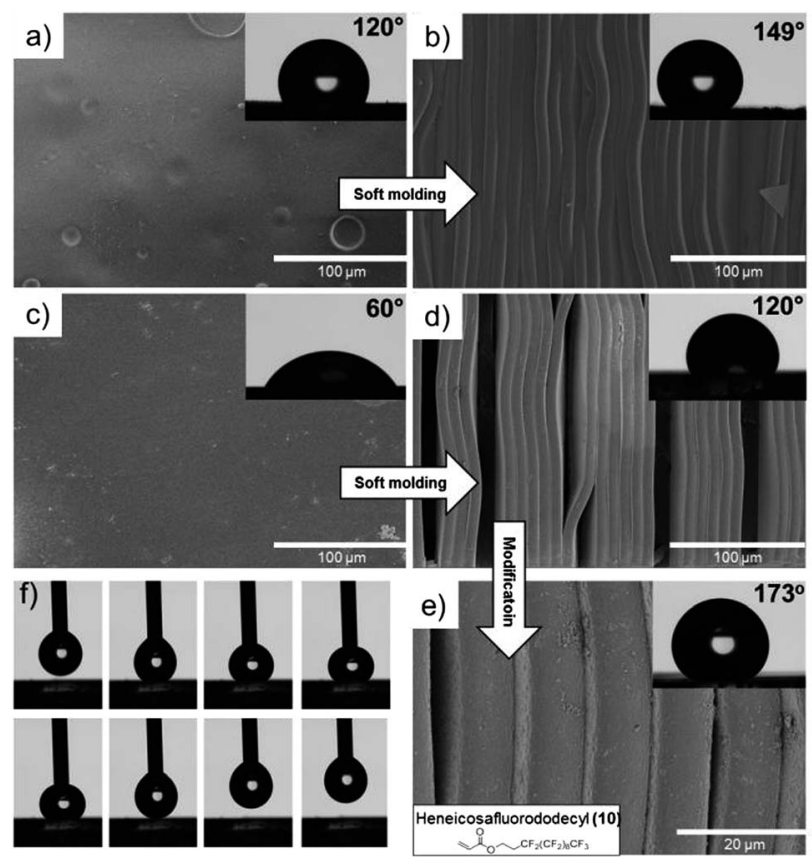

Fig. 6 The effects of surface roughness and postfunctionalization on functional surfaces shown by contact angle measurements and SEM images. ( $a$ and b) Flat and structured soft PDMS network S3; ( $c$ and d) flat and structured rigid organic network R5; (e) facile postfunctionalization of R5 to obtain superhydrophobic surface properties; (f) 1 second snapshots of water rejection of the superhydrophobic substrate. 
As can be seen in Fig. 6f, a 8 second picture sequence showcases the superhydrophobicity making these surfaces ideal as water repellent rigid substrates (see video $\mathrm{S} 1 \dagger$ for the superhydrophobic water repellence surface with 5 and $10 \mu$ water droplets).

In summary, we herein describe a modular approach that exploits the highly efficient and UV initiated thiol-ene coupling chemistry for the fabrication of functional network templates with controllable mechanical properties. By carefully choosing the components and their off-stoichiometric feed, thiol and allyl, functional 3D networks were efficiently constructed under less than 1 second UV exposure at wavelengths of 320-390 nm. The window of Young's modulus obtained ranges from $780 \mathrm{MPa}$ for rigid organic networks to $18 \mathrm{kPa}$ for hydrogel systems. The fabricated networks were further utilized as reactive templates for the functionalization with several vinylic molecules including colorimetric agent to visually confirm successful covalent attachment to the readily available thiol groups. Additionally, the wettability of the networks was manipulated by the introduction of hydrophilic compounds such as PEGallylated chains and hydrophobic fluorinated groups. The introduction of surface roughness through microfabrication coupled with facile postfunctionalizations of fluorinated substituents increased the contact angle of a rigid organic substrate from $60^{\circ}$ to superhydrophobic $173^{\circ}$.

\section{Acknowledgements}

The authors are financially supported by AB WILH. BECKERS Jubileumsfond and Swedish Research Council (grant 2010-453). Dr Fredrik Carlborg (Micro- and Nanosystems, KTH Royal Institute of Technology) is acknowledged for providing silicon master used in microfabrication.

\section{Notes and references}

1 A. L. Hillberg, K. R. Brain and C. J. Allender, Adv. Drug Delivery Rev., 2005, 57, 1875-1889.

2 M. T. Frey, A. Engler, D. E. Discher, J. Lee, Y. L. Wang, E. D. Yu-Li Wang and E. D. Dennis, in Methods in Cell Biology, Academic Press, 2007, pp. 47-65.

3 A. G. Mayes and M. J. Whitcombe, Adv. Drug Delivery Rev., 2005, 57, 1742-1778.
4 X. Y. Qin Dong and G. M. Whitesides, Nat. Protoc., 2010, 5, 491-502.

5 A. Mata, A. Fleischman and S. Roy, Biomed. Microdevices, 2005, 7, 281-293.

6 S. K. Sia and G. M. Whitesides, Electrophoresis, 2003, 24, 3563-3576.

7 J. M. K. Ng, I. Gitlin, A. D. Stroock and G. M. Whitesides, Electrophoresis, 2002, 23, 3461-3473.

$8 \mathrm{~J}$. Zhou, D. A. Khodakov, A. V. Ellis and N. H. Voelcker, Electrophoresis, 2012, 33, 89-104.

9 H. Hillborg and U. W. Gedde, IEEE Trans. Dielectr. Electr. Insul., 1999, 6, 703-717.

10 J. C. McDonald and G. M. Whitesides, Acc. Chem. Res., 2002, 35, 491-499.

11 D. Bartolo, G. Degre, P. Nghe and V. Studer, Lab Chip, 2008, 8, 274-279.

12 C. E. Hoyle and C. N. Bowman, Angew. Chem., Int. Ed., 2010, 49, 1540-1573.

13 A. F. Jacobine, in Radiation Curing in Polymer Science and Technology, ed. J. F. R. Jean-Pierre Fouassier, Elsevier, Essex, England, 1993.

14 C. F. Carlborg, T. Haraldsson, K. Oberg, M. Malkoch and W. van der Wijngaart, Lab Chip, 2011, 11, 3136-3147.

15 M. I. Montañez, Y. Hed, S. Utsel, J. Ropponen, E. Malmström, L. Wågberg, A. Hult and M. Malkoch, Biomacromolecules, 2011, 12, 2114-2125.

16 N. Gupta, B. F. Lin, L. M. Campos, M. D. Dimitriou, S. T. Hikita, N. D. Treat, M. V. Tirrell, D. O. Clegg, E. J. Kramer and C. J. Hawker, Nat. Chem., 2012, 4, 424.

17 L. M. Campos, K. L. Killops, R. Sakai, J. M. J. Paulusse, D. Damiron, E. Drockenmuller, B. W. Messmore and C. J. Hawker, Macromolecules, 2008, 41, 7063-7070.

18 S. Hansson, P. Antoni, H. Bergenudd and E. Malmstrom, Polym. Chem., 2011, 2, 556-558.

19 P. Eyer, F. Worek, D. Kiderlen, G. Sinko, A. Stuglin, V. Simeon-Rudolf and E. Reiner, Anal. Biochem., 2003, 312, 224-227.

20 C. E. Hoyle, A. B. Lowe and C. N. Bowman, Chem. Soc. Rev., 2010, 39, 1355-1387.

21 Y. Xia and G. M. Whitesides, Angew. Chem., Int. Ed., 1998, 37, 550-575.

22 I. P. Parkin and R. G. Palgrave, J. Mater. Chem., 2005, 15, 1689-1695. 